\title{
Predictors of Complete Early Virological Response to Pegylated Interferon and Ribavirin in Egyptian Patients with Chronic Hepatitis C Genotype 4
}

\author{
Gamal El Din Esmat Gamil ${ }^{1}$, Maissa El Said El Raziky ${ }^{1}$, Rabab Maamoun Salama ${ }^{1}$, \\ Wafaa Ahmed El Akel ${ }^{1}$, Waleed Fouad Fathalah ${ }^{1 *}$, Dina Ismail Attia ${ }^{2}$ \\ ${ }^{1}$ Endemic Medicine and Hepatology Department, Faculty of Medicine, Cairo University, Cairo, Egypt; ${ }^{2}$ Endemic Medicine and \\ Hepatology Department, Faculty of Medicine, Beni-Suef University, Beni Suef, Egypt. \\ Email: *walfou2000@gmail.com
}

Received March $1^{\text {st }}, 2013$; revised April $9^{\text {th }}, 2013$; accepted May $9^{\text {th }}, 2013$

Copyright (C) 2013 Gamal El Din Esmat Gamil et al. This is an open access article distributed under the Creative Commons Attribution License, which permits unrestricted use, distribution, and reproduction in any medium, provided the original work is properly cited.

\begin{abstract}
We aim to determine the baseline factors associated with partial and cEVR by analyzing the data of 1861 Egyptian patients treated for 12 weeks with a course of Peg-IFN plus RBV. Base line data of 1861 Egyptian patients with chronic hepatitis $\mathrm{C}$ coming at Cairo-Fatemic Hospital for HCV treatment were studied including full clinical, Ultrasonographic examination, laboratory evaluation and liver biopsy. The most significant variables in relation to complete early virological response were low $\mathrm{Hb}$ level $(<13 \mathrm{gm} / \mathrm{dl})$ with $p<0.01$, the stage of fibrosis $p$ value $<0.05$ and the grades of inflammation $p$ value $<0.05$ were associated with less achievement of c EVR. We conclude that identifying the most significant predictors of response such as $\mathrm{Hb}$, stage of fibrosis $\mathrm{F}$, at baseline before initiating treatment is mandatory to predict which patient will be more expected to achieve a cEVR and thus reducing the side-effects and healthcare costs associated with interferon therapies.
\end{abstract}

Keywords: Predictors of Response; cEVR; HCV Treatment; Peg IFN/Ribavirin

\section{Introduction}

Chronic hepatitis $\mathrm{C}$ is endemic in most regions of the world though prevalence rates vary widely. Over 170 million people are infected with HCV worldwide (about $3 \%$ of the global population). Egypt is among the world's highest prevalence rates of HCV $(10 \%-15 \%$ having HCV antibodies in rural area) [1]. HCV is a major cause of chronic hepatitis, liver cirrhosis and hepatocellular carcinoma and represents the most common cause of liver transplantation in the US and Europe [2].

The current and future burden of disease caused by viral hepatitis in Egypt is significant: it is not an exaggeration to say that viral hepatitis (particularly HCV) is currently and will remain for some time Egypt's most pressing public health issue [3].

PEG-IFN has become the cornerstone of therapy as it extends the duration of therapy and reduces adverse effects and when combined with Ribavirin, the rate of sustained virological response (SVR) has dramatically im-

${ }^{*}$ Corresponding author. proved even in patients with high HCV RNA level $[4,5]$.

Egyptians being treated for $\mathrm{HCV}$ receive 48 weekly subcutaneous doses of pegylated interferon with twice daily doses of Ribavirin. Several specificities of the Egyptian epidemic are to be noted. First, nearly all Egyptian HCV infections (upwards of 95\%) are genotype 4. While HCV genotype has no impact on the course of the disease, different genotypes do react differently to treatment; genotype 4 has an intermediate resistance to treatment. For this reason, Egyptian patients must undergo longer courses of treatment: 48 weeks instead of the 24 weeks recommended [1].

Given the significant side-effects and healthcare costs associated with interferon therapies, identifying patients who are less likely to respond is highly desirable to predict the rate of achieving SVR in the individual patient, before initiating treatment. Patients who failed to achieve a complete early virologic response (cEVR) (defined as undetectable HCV RNA after 12 weeks of treatment) or a partial EVR ( $p$ EVR, defined as $a \geq 2-\log _{10}$ decrease 
from baseline in HCV RNA after 12 weeks of treatment) had a lower likelihood of achieving SVR with an additional 36 weeks of treatment [5-7].

Both virus and host-related elements have been reported as factors correlated to therapeutic effects of combination therapy. A particular focus has been placed on age, gender, Body mass index (BMI), degree of inflammatory activity and fibrosis of the liver and HCV RNA level, HCV genotype and hematological parameters [810]. However many other factors need to be studied.

Identifying these factors may provide information to optimize and/or individualize the treatment of $\mathrm{HCV}$ genotype 4 infected patients, thus improving antiviral response. This is of particular importance because these difficult to treat populations have a high prevalence of $\mathrm{HCV}$ infection and comprise a large proportion of the $\mathrm{HCV}$-infected population in Egypt.

The aim of this study is to analyze the data of 1861 Egyptian patients treated for 12 weeks with a course of Peg-IFN plus RBV, to examine the baseline factors associated with partial and cEVR.

\section{Patients and Methods}

We studied retrospectively the data of 1861 Egyptian patients with chronic hepatitis $\mathrm{C}$ who received antiviral therapy (pegylated-interferon alpha plus Ribavirin) at Cairo-Fatemic Hospital, Ministry of Health and Population, Cairo, Egypt from 2007-2010. Data were retrieved from their medical records after obtaining patient's informed consent and the approval of IRB of the $\mathrm{MOH}$, Patients had code numbers to respect privacy policy. Patients received either pegylated interferon alpha $2 a$ (180 ug once weekly) or pegylated interferon alpha $2 b(1.5$ ug/kg weekly) SC in addition to Ribavirin dose (13 - 15 $\mathrm{mg} / \mathrm{kg} /$ day). Early virologic response (EVR) was assessed after 12 weeks of therapy and patients were categorized into complete EVR, partial EVR or non-EVR $\left(<2-\log _{10}\right.$ decrease from baseline in HCV RNA after 12 weeks of treatment).

Data studied included full clinical examination, ultrasonographic examination and laboratory evaluation (haematological tests, Liver function tests and PCR for detection of HCV viraemia); liver biopsy was done for all patients prior to treatment to assess the grade of inflammation and the stage of fibrosis and to decide whether included or excluded from treatment.

Patients were included according to the national guidelines of National Committee, being adult, naïve, non obese(BMI LESS $<35$ ) of both sexes with no co-morbid conditions and -ve HBsAg, having no contraindications for IFN/RBV therapy and with elevated liver enzymes or normal liver enzymes but with METAVIR score $\geq A 2$ and $F \geq F 2$.
APRI was done and was calculated at a cut off value $<$ $0.8[11]$.

\section{Statistical Analysis}

The SPSS software version 11 was used for data management and analysis. Quantitative data were presented as mean $+\mathrm{SD}$. Qualitative data were presented as frequencies and percentages. For comparison of 3 group's means, Post Hoc test was used. To study the relationship between two variables Spearman's correlation coefficient was calculated. All tests were two tailed and considered statistically significant when ( $p$ value $<0.05$ ).

\section{Results}

Among the studied 1861 patients, 860 received peg interferon alpha2-a while, 972 received peg interferon alpha 2-b in addition to Ribavirin for a period of 12 weeks. They were evaluated for EVR, Partial and non virological response by quantitative testing their HCV RNA by PCR techniques. At 12 weeks, among 1861 patients, 1331 (71.5\%) achieved c EVR, 85 (4.6\%) achieved partial EVR however 445 (23.9\%) were non responders. On analyzing the demographic features shown in (Table 1); of 1861 patients, 1601 were males, 260 were females. Among males 1163 achieved c EVR, and among females 168 achieved c EVR, with a statistically significant difference $<0.05$ between males and females denoting that gender plays a role as a predicting factor for EVR in chronic hepatitis $\mathrm{C}$ patients, however this statistical difference may be due to the larger number of males included in the study compared to females, on doing Univariate analysis of predictors of cEVR, male gender was associated with failure of cEVR with a statistically significant difference. Regarding the base line characteristics, biochemical and hematological findings of our patients, as the age, BMI, the albumin, the AST, ALT \& ALP, the total and direct bilirubin, these parameters were of no significant impact on cEVR, however the $\mathrm{Hb}$ level was higher (mean 13.7 and $14.1 \mathrm{gm} / \mathrm{dl}$ ) in patients who achieved a cEVR and partial EVR compared to non responders who have lower $\mathrm{Hb}$ level (mean $12.5 \mathrm{gm} / \mathrm{dl}$ ) with a highly statistical significant difference $<0.001$. The alpha feto-protein was significantly higher in non responders than in patients with cEVR with a statistical significance $<0.05$, denoting that the higher the alpha fetoprotein level the lesser the chance to achieve a cEVR.

On doing Univariate analysis of these predictors, $\mathrm{Hb}<$ 13 was a predictor of failure of achievement of cEVR with a statistical significance $<0.01$ in both uni- and multivariate analysis. Eighty one \% of patients with $\mathrm{HCV}$ RNA less $<800$ IU achieved c EVR compared to $19 \%$ and $4 \%$ with no EVR or partial EVR respectively with a statistical significant difference $<0.05$. 
Table 1. The baseline variables in relation to the achievement of EVR.

\begin{tabular}{|c|c|c|c|c|}
\hline Variables & $\begin{array}{c}\mathrm{cEVR} n= \\
1331(71 \%)\end{array}$ & $\begin{array}{c}\text { Non EVR } \\
\mathrm{n}=445(24 \%)\end{array}$ & $\begin{array}{l}\text { Partial EVR n } \\
\quad=85(5 \%)\end{array}$ & $P$ \\
\hline \multicolumn{5}{|c|}{ Gender } \\
\hline Male & 1163 & 363 & 75 & \multirow{2}{*}{0.008} \\
\hline Female & 168 & 82 & 10 & \\
\hline Age (years) & $41.3 \pm 9.7$ & $42.1 \pm 9.7$ & $42.7 \pm 9.1$ & 0.08 \\
\hline BMI $\left(\mathrm{kg} / \mathrm{m}^{2}\right)$ & $27.8 \pm 4.0$ & $28.0 \pm 4.2$ & $27.9 \pm 4.2$ & 0.54 \\
\hline ALT folds & $1.6 \pm 1.27$ & $1.57 \pm 1.12$ & $1.39 \pm 0.87$ & 0.087 \\
\hline AST folds & $1.4 \pm 1.1$ & $1.4 \pm 087$ & $1.4 \pm 1.1$ & 0.89 \\
\hline ALP folds & $0.6 \pm 0.6$ & $0.6 \pm 0.4$ & $0.5 \pm 0.3$ & 0.31 \\
\hline Albumin g/dl & $4.2 \pm 0.5$ & $4.2 \pm 0.5$ & $4.2 \pm 0.4$ & 0.53 \\
\hline Total bil. & $0.80 \pm 0.29$ & $0.8150 \pm 0.32$ & 0.8589 & 0.319 \\
\hline $\mathrm{Hb}(\mathrm{g} / \mathrm{dl})$ & $13.7 \pm 2.5$ & $12.5^{*} \pm 4.0$ & $14.1 \pm 2$ & 0.000 \\
\hline $\mathrm{PLT} \times 10^{3} / \mu \mathrm{L}$ & $211.3 \pm 68.9$ & $207.0 \pm 62.5$ & $198.4 \pm 51.7$ & 0.145 \\
\hline $\mathrm{WBC} \times 10^{3} / \mathrm{cm}$ & $6.42 \pm 1.9$ & $6.3 \pm 1.8$ & $6.3 \pm 2.0$ & 0.647 \\
\hline AFP folds & $0.58 \pm 1.3$ & $0.8 \pm 1.56$ & $0.81 \pm 1.42$ & 0.001 \\
\hline \multicolumn{5}{|c|}{ HCV-RNA } \\
\hline$<800$ & $973(81 \%)$ & $216(19 \%)$ & $52(4 \%)$ & \multirow{2}{*}{0.025} \\
\hline$>800$ & $192(74 \%)$ & $43(16 \%)$ & $24(9.2)$ & \\
\hline \multicolumn{5}{|c|}{$\operatorname{Activity(n)}$} \\
\hline A1 & 419 & 107 & 22 & \multirow{3}{*}{0.62} \\
\hline A2 & 489 & 107 & 34 & \\
\hline A3 & 178 & 37 & 14 & \\
\hline \multicolumn{5}{|c|}{ Fibrosis } \\
\hline F1 & 674 & 128 & 38 & \multirow{4}{*}{0.04} \\
\hline $\mathrm{F} 2$ & 210 & 65 & 14 & \\
\hline F3 & 139 & 34 & 10 & \\
\hline $\mathrm{F} 4$ & 75 & 23 & 9 & \\
\hline \multicolumn{5}{|c|}{ IFN Type } \\
\hline Alpha 2a & 650 & 178 & 32 & \multirow{2}{*}{0.004} \\
\hline Alpha 2b & 668 & 251 & 53 & \\
\hline
\end{tabular}

674 patients out of 1331 of c EV responders were F1 compared to only 75 with F4 who achieved c EVR, with a statistical significant difference of $<0.05$. Univariate and multivariate analysis of stages of fibrosis (F2, F3 and F4) with achievement of cEVR shows these stages are associated with failure of cEVR with a statistical significance of $<0.05$ and the higher the stage of fibrosis the greater the failure of cEVR. Regarding the grades of inflammation A, on Univariate analysis, A1, A2 and A3 were associated with cEVR with a statistical significance for $\mathrm{A} 2$ and $\mathrm{A} 3<0.05$ on Univariate and multivariate analysis and for $\mathrm{A} 3$ on multivariate analysis $<0.05$, so A1 and $\mathrm{A} 2$ and $\mathrm{A} 3$ are predictors of achievement of cEVR.

650 patients received PEG IFN alpha 2 a and achieved c EVR compared to 178 who did not respond and 32 with partial response with a statistically significant difference $<0.05$, however 668 patients achieved cEVR were on PEG IFN alpha $2 \mathrm{~b}$ compared to 251 who did not respond and 53 partial responders with no statistical significant difference.

Table 2 shows the relation between APRI cutoff at 0.8 and the achievement of EVR in chronic HCV patients.

$893(69.2 \%)$ patients with APRI $<0.8$ achieved cEVR compared to 398 (30.8\%) patients with APRI > 0.8 however there was no statistical significant difference and among patients with APRI $<0.8,893$ out of 1225 achieved c EVR compared to 276 with no EVR and 56 with partial response with no statistical significant difference, in univariate analysis APRI at 0.8 cutoff value was associated with failure of cEVR but with no statistical significance.

As a conclusion, in logistic regression analysis (Table 3), Univariate analysis was done among all variables and then multivariate analysis was done among the most significant variables in relation to complete early virological response; by univariate analysis achievement of cEVR was related to higher stages of fibrosis, grades A2 and $\mathrm{A} 3$ of inflammation and $\mathrm{Hb}<13 \mathrm{mg} / \mathrm{dl}$.

By multivariate analysis, cEVR showed significant association with higher fibrosis stages (F2, F3 and F4) and grades of inflammation (A2 and A3), low Hb level $(<13 \mathrm{gm} / \mathrm{dl})$ with a $p$ value $<0.05$, denoting that these are the most significant predictors of response useful to be known at the baseline before initiating treatment to predict which patient will be more expected to achieve a complete early virological response and thus reducing the side-effects and healthcare costs associated with interferon therapies.

\section{Discussion}

$\mathrm{HCV}$ is a major cause of chronic hepatitis, liver cirrhosis and hepatocellular carcinoma. Given the significant sideeffects and healthcare costs associated with interferon therapies, this retrospective study was conducted, on evaluating the predictors of early virological response at the base line before initiating treatment, among 1861 patients with chronic hepatitis $\mathrm{C}$ of genotype 4 , we demon-

Table 2. Relation between APRI cutoff at 0.8 and the achievement of EVR in chronic HCV patients.

\begin{tabular}{cccc}
\hline \multirow{2}{*}{ APRI } & \multicolumn{3}{c}{ EVR groups } \\
\cline { 2 - 4 } & $\mathrm{cEVR} \mathrm{n}(\%)$ & Non-EVR n(\%) & $p$ EVR n(\%) \\
\hline$<0.8(\mathrm{n}=1225)$ & $893(69.2 \%)$ & $276(63.6 \%)$ & $56(69.1 \%)$ \\
$>0.8(\mathrm{n}=581)$ & $398(30.8 \%)$ & $158(36.4 \%)$ & $25(30.9 \%)$ \\
\hline
\end{tabular}


Table 3. The logistic regression analysis of variables in relation to EVR.

\begin{tabular}{|c|c|c|c|c|c|}
\hline \multirow{2}{*}{ Variables } & \multicolumn{3}{|c|}{ Univariate analysis } & \multicolumn{2}{|c|}{ Multivariate analysis } \\
\hline & OR & $\mathrm{P}$ & CI & OR & $\mathrm{P}$ \\
\hline Age $>40$ & 0.8 & 0.26 & $0.58-1.1$ & 0.82 & 0.23 \\
\hline Male gender & 1.2 & 0.43 & $0.7-1.9$ & & \\
\hline $\mathrm{BMI}>30$ & 1.1 & 0.44 & $0.8-1.6$ & & \\
\hline APRI at 0.8 & 1.1 & 0.33 & $0.73-1.4$ & & \\
\hline HCV RNA $>800$ & 0.8 & 0.34 & $0.5-1.2$ & & \\
\hline $\mathrm{AFP}>1$ & 1.2 & 0.52 & $0.7-1.9$ & & \\
\hline \multicolumn{6}{|c|}{ Stages of fibrosis } \\
\hline $\mathrm{F} 2$ & 1.8 & 0.007 & $1.2-2.7$ & 1.9 & 0.02 \\
\hline F3 & 1.9 & 0.02 & $1.1-3.2$ & 2.0 & 0.01 \\
\hline F4 & 2.0 & 0.048 & $1-4.1$ & 2.0 & 0.03 \\
\hline \multicolumn{6}{|c|}{ Grades of activity } \\
\hline A1 & 0.61 & 0.01 & $0.4-0.9$ & 0.6 & 0.007 \\
\hline $\mathrm{A} 2$ & 0.4 & 0.002 & $0.2-0.7$ & 0.4 & 0.004 \\
\hline $\mathrm{A} 3$ & 0.3 & 0.36 & $0.03-3.3$ & 0.4 & 0.004 \\
\hline $\mathrm{HB}<13$ & 2.3 & 0.00 & $1.7-3.3$ & 2.2 & 0.00 \\
\hline IFN $\alpha-2 b$ & 1.3 & 0.087 & $0.96-1.8$ & 1.3 & 0.07 \\
\hline
\end{tabular}

OR: odds ratio; CI: confidence interval.

strated that at 12 weeks of treatment with Pegasys and Ribavirin or Peg IFN and Ribavirin, 1331 (71.5\%) achieved complete EVR, 85(4.6\%) were partial responders and 445(23.9\%) didn't respond to treatment. Our results were comparable with Torres and colleagues [12] who evaluated factors associated with rapid and early virologic response to peg interferon alfa-2a/Ribavirin treatment in HCV genotype 1 patients representative of the general chronic hepatitis $\mathrm{C}$ population and found that out of the 1550 patients treated with peg interferon alfa2a plus Ribavirin, 242 (15.6\%) patients achieved RVR and $837(54.0 \%)$ which was lower than our results. Several predictive factors play a significant role in the achievement of EVR. In our study the response in males was significantly higher than females, Martinot-Pegnoux and co-workers [13] reported that females respond better than males but this difference in our study may be due the larger number of males included compared to females as well as the difference in the type of interferon received as treatment.

Regarding the $\mathrm{Hb}$ level in our study and its impact on EVR, it was higher (mean 13.7 and $14.1 \mathrm{gm} / \mathrm{dl}$ ) in patients who have achieved a cEVR and $p$ EVR compared to non responders who have lower $\mathrm{Hb}$ level (mean 12.5 $\mathrm{gm} / \mathrm{dl}$ ) with a highly statistical significant difference $<$ 0.001 , showing that the $\mathrm{Hb}$ level is one of the important predictive factor of EVR response and thus of SVR, this may be explained by the fact that these patients with a higher HB might need less frequent dose reduction of INF and/or RBV than those with lower base line HB and maintenance of higher doses of both is related to better response. In the study conducted by Torres et al., [12] the decrease of $\mathrm{Hb}$ from baseline to week 12 in the cEVR group was greater than that in the non-cEVR group $(p=$ $0.0160)$.

The alpha feto protein was also found to be one of the predictors of response being significantly higher in non responders than in those with $C$ EVR with a statistical significance $<0.05$, denoting that the higher the alpha fetoprotein level the lesser the chance to achieve a $\mathrm{C}$ EVR. Abdo and Sanai [14] cited that a higher AFP level is associated with a negative treatment outcome in chronic hepatitis $\mathrm{C}$ patients of genotype 4. Similar findings have been found in genotype 1 patients [8].

The HCV RNA level was a good predictor of response in our study as patients with an HCV RNA level $<600$ $\mathrm{IU} / \mathrm{ml}$ prior to treatment achieved a complete EVR with a statistical significant difference compared to patients with partial response and non responders, however it is therefore difficult to predict the virological response solely from the amount of HCVRNA before starting the treatment [15].

In our study, the type of interferon plays a statistically significant role in patients with Non EVR and with partial EVR as the early response to treatment is significantly lower with PEG-INT compared to PEGASYS ( $p<$ $0.004)$. This finding was similar to two studies (including 100 and 38 patients, respectively) using the alfa $2 a$ form of pegylated interferon in genotype 4 patients and suggested that the response rates may be higher with Pegasys [16,17], as well as, In a study of Ribavirin in combination with either PEG-IFN alfa-2b or PEG-IFN alfa-2a for the treatment of chronic HCV infection, Ascione et al. [18] reported a higher SVR rate with PEGIFN alfa-2a than with PEG-IFN alfa-2b $(68 \%$ versus $54.4 \%$ ). However, any attempt at comparing treatment success between the two forms of pegylated interferons among this particular genotype would be unfair and premature, due to the lack of large, prospectively conducted studies using the Alfa $2 a$ form of pegylated interferon [14].

One of the most important predictors of EVR in our study is the stage of fibrosis, as the higher the stage of fibrosis, the lower the achievement of a C EVR, our finding was also reported by Torres et al. [12] who demonstrated, that one of the independent factors associated with a cEVR was the non-cirrhotic status of the patients HCV I at baseline. De Careaga [19] reported that patients with hepatitis $\mathrm{C}$ and cirrhosis have lower rates of sustained responses even with the absence of cirrhosis, the 
degree of response to treatment decreases with the increase of stage of fibrosis.

Although not statistically significant, we found that the lower the APRI the better the achievement of a C EVR, however Mata-Marin et al.; [20] found no association when APRI was more than 1.2 and early virological response when stratifying between genotype 1 and genotype other than 1 to evaluate APRI as predictor of EVR.

Previous studies have not explored the utility of noninvasive tests to assess liver fibrosis for the prediction of viral response in hepatitis $C$ naive patients. In our study, we believe that our results show that APRI is a predictor of early viral response in $\mathrm{HCV}$ naive patients.

From our study we can conclude that proper evaluation of the predictors of $\mathrm{c}$ EVR such as $\mathrm{Hb}$ level, stage of fibrosis and grade of inflammation, PCR, Alpha fetoprotein, APRI and the type of interferon better to be used at the base line before initiating treatment is important in determining the possibility of achievement of c EVR and hence SVR and also in reducing the burden of cost and the side effects of chronic HCV treatment for patients who are not likely to achieve neither c EVR nor SVR.

\section{Acknowledgements}

Special thanks to Cairo Fatemic Hospital and the ministry of Health, for their active participation in the practical part of this work and their active cooperation in giving the permission for the collection of data of chronic HCV patients who were subjected to proper evaluation and treatment of $\mathrm{HCV}$.

\section{REFERENCES}

[1] Egyptian National Control Strategy for Viral Hepatitis, 2008-2012.

[2] G. M. Lauer and B. D. Walke, "Hepatitis C Virus Infection," The New England Journal of Medicine, Vol. 345, 2001, pp. 41-52. doi:10.1056/NEJM200107053450107

[3] K. W. Ray, "Global Epidemiology and Burden of Hepatitis C," Microbes and Infection, Vol. 4, No. 12, 2002, pp. 1219-1225. doi:10.1016/S1286-4579(02)01649-0

[4] J. Stephanos, S. Hoel Jr., R. Timothy, D. Vijayan, et al., "Peginterferon- $\alpha 2 \mathrm{a}$ and Ribavirin Combination Therapy in Chronic Hepatitis C: A Randomized Study of Treatment Duration and Ribavirin Dose," Annals of Internal Medicine, Vol. 140, No. 5, 2004, pp. 346-355. doi:10.7326/0003-4819-140-5-200403020-00010

[5] M. P. Manns, J. G. McHutchison, S. C. Gordon, V. K. Rustgi, et al., "Peginterferon Alfa-2b plus Ribavirin Compared with Interferon Alfa-2b plus Ribavirin for Initial Treatment of Chronic Hepatitis C: A Randomised Trial," Lancet, Vol. 358, No. 9286, 2001, pp. 958-965. doi:10.1016/S0140-6736(01)06102-5

[6] S. S. Lee, E. J. Heathcote, K. R. Reddy, S. Zeuzem, et al.,
"Prognostic Factors and Early Predictability of Sustained Viral Response with Peginterferon Alfa-2a (40KD)," Journal of Hepatology, Vol. 37, No. 4, 2002, pp. 500-506. doi:10.1016/S0168-8278(02)00211-8

[7] G. L. Davis, J. B. Wong, J. G. McHutchison, M. P. Manns, et al., "Early Virologic Response to Treatment With Peginterferon Alfa-2b plus Ribavirin in Patients with Chronic Hepatitis C," Hepatology, Vol. 38, No. 3, 2003, pp. 645652. doi:10.1053/jhep.2003.50364

[8] N. Akuta, F. Suzuki, Y. Kawamura, H. Yatsuji, et al., "Predictive Factors of Early and Sustained Responses to Peginterferon plus Ribavirin Combination Therapy in Japanese Patients Infected with Hepatitis C Virus Genotype 1b: Amino Acid Substitutions in the Core Region and Low-Density Lipoprotein Cholesterol Levels," Journal of Hepatology, Vol. 46, No. 3, 2007, pp. 403-410. doi:10.1016/j.jhep.2006.09.019

[9] M. W. Fried, M. L. Shiffman, K. R. Reddy, et al., "Peginterferon Alfa-2a plus Ribavirin for Chronic Hepatitis C Virus Infection," The New England Journal of Medicine, Vol. 347, No. 13, 2002, pp. 975-982. doi:10.1056/NEJMoa020047

[10] K. L. Lindsay, C. Morishima, E. C. Wright, J. L. Dienstag, et al., "Blunted Cytopenias and Weight Loss: New Correlates of Virologic Null Response to Re-Treatment of Chronic Hepatitis C," Clinical Gastroenterology and Hepatology, Vol. 6, No. 2, 2008, pp. 234-241. doi:10.1016/j.cgh.2007.11.020

[11] C.-T. Wai, J. K. Greenson, R. J. Fontana, J. D. Kalbfleisch, et al., "A Simple Noninvasive Index Can Predict Both Significant Fibrosis and Cirrhosis in Patients with Chronic Hepatitis C," Hepatology, Vol. 38, No. 2, 2003, pp. 518-526. doi:10.1053/jhep.2003.50346

[12] M. Rodriguez-Torres, M. S. Sulkowski, R. T. Chung, F. M. Hamzeh and D. M. Jensen, "Factors Associated with Rapid and Early Virologic Response to Peginterferon Alfa2a/Ribavirin Treatment in HCV Genotype 1 Patient's Representative of the General Chronic Hepatitis C Population," Journal of Viral Hepatitis, Vol. 17, No. 2, 2010, pp. 139-147. doi:10.1111/j.1365-2893.2009.01157.x

[13] M. Martinot-Peignoux, N. Boyer, M. Pouteau, C. Castelnau, et al., "Predictors of Sustained Response to Alpha Interferon Therapy in Chronic Hepatitis C," Journal of Hepatology, Vol. 29, No. 2, 1998, pp. 214-223. doi:10.1016/S0168-8278(98)80006-8

[14] A. A. Abdo and F. M. Sanai, "Predictors of Sustained Virologic Response in Hepatitis C Genotype 4: Beyond the Usual Suspects," Annals of Saudi Medicine, Vol. 29, 1, 2009, pp. 1-3. doi:10.4103/0256-4947.51810

[15] I. Namiki, A. Yasuhiro and K. Masayuki, "Predictors of Virological Response to a Combination Therapy with Pegylated Interferon plus Ribavirin Including Virus and Host Factors," Hepatitis Research and Treatment, Vol. 2010, 2010, 7 Pages.

[16] S. Males, R. R. Gad, G. Esmat and H. Abobakr, "Serum Alpha-Foetoprotein Level Predicts Treatment Outcome in Chronic Hepatitis C," Antiviral Therapy, Vol. 12, No. 5, 2007, pp. 797-803. 
[17] M. F. Derbala, S. R. Al Kaabi, N. Z. El Dweik and F. Passic, "Treatment of Hepatitis C Virus Genotype 4 with Peginterferon Alfa-2a: Impact of Bilharziasis and Fibrosis Stage," World Journal of Gastroenterology, Vol. 12, No. 35, 2006, pp. 5692-5698.

[18] A. Ascione, M. De Luca, M. T. Tartaglione, F. Lampasi, et al., "Peginterferon Alfa-2a plus Ribavirin Is More Effective than Peginterferon Alfa-2b plus Ribavirin for Treating Chronic Hepatitis C Virus Infection," Gastroenterology, Vol. 138, No. 1, 2010, pp. 116-122. doi:10.1053/j.gastro.2009.10.005
[19] B. O. De Careaga, "Predictive Factors for Response to Treatment of Chronic Hepatitis C," Annals of Hepatology, Vol. 5, Suppl. 1, 2006, pp. s24-s28.

[20] J. A. Mata-Marín, J. L. Fuentes-Allen, J. Gaytán-Martínez, B. Manjarrez-Téllez, et al., "APRI as a Predictor of Early Viral Response in Chronic Hepatitis C Patients," World Journal of Gastroenterology, Vol. 15, No. 3, 2009, pp. 4923-4927. doi:10.3748/wig. 15.4923 